Relations industrielles

Industrial Relations

\title{
Les Canadiens et les nouveaux développements industriels
}

\section{Roger Vézina}

Volume 13, numéro 2, avril 1958

URI : https://id.erudit.org/iderudit/1022441ar

DOI : https://doi.org/10.7202/1022441ar

Aller au sommaire du numéro

Éditeur(s)

Département des relations industrielles de l’Université Laval

ISSN

0034-379X (imprimé)

1703-8138 (numérique)

Découvrir la revue

Citer ce document

Vézina, R. (1958). Les Canadiens et les nouveaux développements industriels. Relations industrielles / Industrial Relations, 13(2), 162-165.

https://doi.org/10.7202/1022441ar
Résumé de l'article

Voici la première partie d'un exposé présenté par M. Roger Vézina, directeur général de la Chambre de Commerce de Québec, lors de l'inauguration des séances d'études économiques du Jeune Commerce, le 25 février 1958, à l'Université Laval.
Tous droits réservés @ C Département des relations industrielles de l’Université Laval, 1958
Ce document est protégé par la loi sur le droit d'auteur. L’utilisation des services d'Érudit (y compris la reproduction) est assujettie à sa politique d'utilisation que vous pouvez consulter en ligne.

https://apropos.erudit.org/fr/usagers/politique-dutilisation/ 


\section{Les Canadiens et les nouveaux développements industriels}

\section{Roger VÉZINA}

Voici la première partie d'un exposé présenté par M. Roger Vézina, directeur général de la Chambre de Commerce de Québec, lors de linanguration des séances d'études économiques du Jeune Commerce, le 25 février 1958, à l'Université Laval.

Une des principales caractéristiques de l'industrie canadienne, principalement celle d'après-guerre, c'est d'ètre de moins en moins canadienne et de plus en plus américaine et britannique.

Devons-nous blâmer les Américains et les Britanniques de manifester plus d'esprit d'initiative, de perspicacité et de confiance dans l'avenir de l'économie canadienne que les Canadiens eux-mèmes? Je ne le crois pas. Je crois qu'il importe plutôt d'éveiller la conscience collective des Canadiens en leur démontrant qu'ils pratiquent une politique de placements qui les rend trop créanciers, rentiers de l'Etat et des corps publics ou privés, et pas assez propriétaires des entreprises vraiment profitables, c'est-à-dire de celles qui extraient ou transforment les immenses ressources naturelles du pays.

Presque la moitié des capitaux étrangers placés au Canada au cours du siècle actuel est entrée depuis la fin de la seconde guerre mondiale, et la plus grande partie après 1948. Depuis dix ans, $\$ 8$ milliards de capitaux étrangers ont été placés au Canada et les placements ainsi faits dépassent présentement les $\$ 15$ milliards. Les capitaux américains comptent pour les trois quarts de ce total et de l'augmentation, et les britanniques, pour moins d'un cinquième.

Présentement, l'étranger possède ou contrôle l'industrie pétrolière canadienne dans une proportion de 70 p.c., l'industrie minière dans une proportion de 59 p.c., et l'industrie manufacturière, autre que le raffinage du pétrole, dans une proportion de 46 p.c.

$\mathrm{Si}$, depuis dix ans, exprimé en dollars constants, le rythme des immobilisations a quadruplé, la valeur brute des produits fabriqués a plus que doublé, la production minérale a triplé; si, depuis dix ans, le Canada est devenu la sixième puissance industrielle au monde, prenant place avant l'Italie et le Japon, il nous faut reconnaître bien humblement que les Canadiens et leurs institutions financières ne sont pas seuls responsables d'une telle réussite et qu'une grande part des mérites revient à l'esprit d'initiative de nos voisins du Sud et des Britanniques.

Parmi les raisons qui empêchent les Canadiens de prendre une part plus active dans les nouveaux développements industriels du pays, 
il faut mentionner:

1.-Le manque de préparation technologique et l'insuffisance de la recherche et du perfectionnement scientifiques;

2.-L'existence de lois beaucoup trop prohibitives et rigides et qui ne tiennent pas assez compte de la réalité économique en matière de placement;

3.-Le siphonnage systématique de l'épargne canadienne par les emprunts publics.

Il est évident que les Canadiens sont beaucoup plus les bénéficiaires que les initiateurs de l'automatisme et, d'une façon générale, de l'amélioration constante des techniques de production. N'étant pas propriétaires des nouvelles découvertes, notamment celle de l'industrie pétro-chimique qui progresse à vue d'oeil, nous ne sommes généralement pas non plus propriétaires des industries qui les exploitent.

Personne ne contestera que dans l'industrie moderne les sommes qui peuvent être affectées à la recherche, mème à la recherche pure, demeurent le plus fructueux placement pour un pays. Ici, nous devons accuser insuffisance, sinon carence complète dans certains cas.

Présentement, les industries canadiennes dépensent environ $\$ 80$ millions par année pour la recherche et le perfectionnement scientifiques. Sur 38,000 établissements manufacturiers, 318 seulement, c'est-àdire moins de 0.9 p.c., dirigent leurs propres programmes de recherches et de perfectionnement. Or, 235 reçoivent gratuitement les résultats de recherches faites par d'autres et 50 p.c. de ce nombre (probablement les plus importantes entreprises du pays) reçoivent leurs renseignements de sociétés-mères hors du pays. Alors que l'industrie canadienne dépense environ \$5 par habitant pour la recherche, le Royaume-Uni et les Etats-Unis dépensent quatre fois plus. Définitivement, nous sommes ici en présence d'une des raisons les plus importantes du manque de participation de Canadiens dans le mouvement d'industrialisation rapide de notre pays.

En vue de l'élaboration d'un programme de recherche et de perfectionnement scientifiques plus agressif et vigoureux, nous croyons que non seulement les industries, mais également les institutions financières canadiennes devraient faire leur part en contribuant, proportionnellement à leur actif, à un fonds commun de recherches. Un tel fonds, exempt d'impôts, serait peut-être leur plus tangible contribution au progrès et à la prospérité du Canada tout entier.

Un autre facteur défavorable: l'existence de lois prohibitives et rigides qui ne tiennent nullement compte de la réalité économique en matière de placements. Les grands réservoirs de l'épargne populaire que sont les banques, caisses et sociétés d'épargne, compagnies de fiducie et compagnies et mutuelles d'assurance-vie et fonds de pension, ne disposent légalement d'aucune sorte d'initiative en matière de placements dits de promotion pour la mise en valeur de nos res- 
sources naturelles. Les lois qui régissent ces institutions sont faites pour la protection farouche de l'épargne par l'acquisition exclusive de valeurs dites de tout repos, pendant que les étrangers éveillés, eux, se portent acquéreurs de l'industrie et d'un sous-sol de tout repos. Ce n'est pourtant pas en consacrant la primauté du rentier par des textes de lois que les Canadiens parviendront à jouer un rôle de premier plan dans le développement industriel d'un pays en plein essor. Ce ne sont pourtant pas non plus les capitaux qui manquent. Ainsi, avec moins de 20 p.c. de son actif, une seule compagnie d'assurance-vie exclusivement canadienne aurait pu assumer la responsabilité financière exclusive de tout le gigantesque développement minier du Nouveau-Québec. Une autre aurait pu assumer celui de la Canadian British Aluminium à Baie Comeau.

Inclinons-nous donc devant l'esprit d'initiative des directeurs de l'Iron Ore et de la C.B.A. et souhaitons qu'un jour nos institutions financières canadiennes aient le pouvoir légal d'en faire autant.

Il est temps, croyons-nous, que la porte des placements s'entrouvre un peu plus et qu'avec la garantie partielle de l'Etat si nécessaire nos institutions financières puissent prendre une part plus active dans le développement industriel et minier du pays. C'est dans cet état d'esprit que la Chambre de Commerce de Québec déclarait en janvier dernier:

«Le Canada a atteint un degré de maturité économique suffisant pour adopter les mesures législatives et bancaires qui s'imposent en vue d'inciter les Canadiens et les institutions financières canadiennes à prendre une part plus active dans le développement et l'exploitation de nos ressources naturelles».

Quant au siphonnage systématique de l'épargne canadienne par les emprunts de l'Etat, il a eu pour résultat de rendre les Canadiens et les institutions financières canadiennes propriétaires de la dette nationale dans une proportion de 90 p.c., pendant que les Américains et les Britanniques devenaient propriétaires des nouvelles entreprises canadiennes dans la même proportion. Bref, par suite de la politique de financement systématique des emprunts de l'Etat sur le marché domestique, les Canadiens sont devenus créanciers, rentiers de l'Etat à 3 et 4 p.c., et les étrangers, propriétaires des nouvelles entreprises privées à 10 p.c. et plus.

S?ns doute, la mise en valeur de nos ressources minérales et le rapide développement industriel d'après-guerre n'ont pu se faire sans entraîner un sérieux déséquilibre de notre balance commerciale provoqué par l'importation d'outillage et de machinerie lourde dont nous ne sommes pas producteurs et qui fut payée, sans compromettre notre balance des paiements, grâce aux placements ou transferts de capitaux des Américains dans leurs entreprises canadiennes. Notons cependant que les Canadiens auraient pu importer les biens de capitaux dont ils 
auraient eu besoin, sans compromettre davantage notre balance des paiements, si l'Etat avait financé une partie de ses emprunts sur le marché étatsunien. Ainsi, les rôles auraient été renversés et les Américains seraient devenus les créanciers obligatoires, pendant que les Canadiens eux, seraient devenus propriétaires des nouvelles entreprises productives.

\section{LE CHÔMAgE CAUSÉ PAR UNE MAUVAISE ORIENTATION}

DE NOTRE POLITIQUE MONÉTAIRE

Mavrice Bouchard

\section{Faits et tendances}

En 1957, du mois d'octobre à décembre, les pourcentages mensuels du nombre de chômeurs par rapport à toute la main-d'oeuvre du Canada ont été deux fois plus élevés que durant la mème période en $1956 .^{1}$

\begin{tabular}{|c|}
\hline Octobre \\
\hline Novembre \\
\hline Décembre \\
\hline Janvier \\
\hline Février \\
\hline Mars \\
\hline
\end{tabular}

Chaque année, à partir de janvier, le nombre de chômeurs s'accroît fortement en raison du fait que l'hiver ralentit les activités dans plusieurs industries. Cette année nous avons toutes les raisons de croire que ce facteur saisonnier joue au moins à l'égal des années passées. Compte tenu, d'autre part, des éléments de crise qui pèsent sur l'ensemble de l'économie depuis un an, il n'est pas exagéré d'affirmer que le chômage actuel, au début de février, constitue une proportion probable de $11 \%$ de la main-d'oeuvre au Canada, soit un nombre approximatif de 650,000 travailleurs. D'ici la fin de mars, il faut s'attendre à dépasser le nombre de 700,000 et la proportion de $12 \%$.

Dans le Québec, où nous comptons environ 28\% de la maind'oeuvre canadienne, nous avons par ailleurs entre 35 et $40 \%$ de tous les chômeurs. Si nous acceptons la proportion de $38 \%$ comme base d'estimation, nous aurions actuellement 247,000 chômeurs environ. Pour la fin de mars, nous devons prévoir approximativement 270,000

(1) Le Bureau fédéral de la Statistique établit le nombre des chômeurs à la première moitié de chaque mois. Cf B.F.S. La main-d'oeuvre. 